\title{
ATTRACTORS IN RESTRICTED CELLULAR AUTOMATA
}

\author{
MIKE HURLEY
}

(Communicated by Kenneth R. Meyer)

\begin{abstract}
The goal of this note is to extend previous results about the dynamics of cellular automata to "restricted cellular automata." Roughly speaking, a cellular automaton is a rule that updates a configuration of "states" that are arranged along the integer lattice in $\mathbb{R}$. In applications one often thinks of one of these states as "blank" or "quiescent," while the other "active" states evolve against a quiescent background. Often the physically relevant configurations are those with only a finite number of active states. If $X_{0}$ is the set of all such states, and if a cellular automaton maps $X_{0}$ to $X_{0}$, then its restriction to $X_{0}$ is a restricted cellular automaton. The main results show that there are rather strong constraints on the collection of attractors for any restricted cellular automaton. These constraints parallel those described in $[\mathrm{H} 1]$ for the unrestricted case.
\end{abstract}

Cellular automata are a class of discrete dynamical systems on a Cantor set $X$. This Cantor set is defined as the bi-infinite product of a finite set $S$ with itself: $X=\prod_{n \in \mathbb{Z}} S$. Giving $S$ the discrete topology and $X$ the product topology makes $X$ a compact space homeomorphic to the usual Cantor middle-third set. One can consider elements of $X$ as functions from $\mathbb{Z}$ to $S$, $X=\{x: \mathbb{Z} \rightarrow S\}$. The shift map is the homeomorphism $\sigma$ on $X$ that is defined by $(\sigma x)(n)=x(n+1)$ for each $n$ in $\mathbb{Z}$ and $x \in X$. A map $f: X \rightarrow X$ is a cellular automaton if it is continuous and commutes with $\sigma: f \circ \sigma=\sigma \circ f$.

The Curtis-Hedlund-Lyndon theorem [Hed] gives a characterization of cellular automata that makes them easy to construct: $f$ is cellular automaton if and only if there is an integer $k \geq 0$ and a map $F: S^{2 k+1} \rightarrow S$ with the property that

$$
(f x)(n)=F(x(n-k), \ldots, x(n+k))
$$

for each $x \in X$ and integer $n$. $F$ is called a block map that generates $f$.

Cellular automata were introduced by von Neumann as a model of biological self-reproduction. Since their introduction, and increasing in recent years, they have been proposed as models in a number of diverse areas (see, e.g., [FTS, W]). The general dynamical behavior of cellular automata is quite complex; in fact, a number of basic questions about cellular automata turn out to be formally undecidable [CHY]. On the other hand the dynamics of cellular automata are

Received by the editors October 13, 1990.

1991 Mathematics Subject Classification. Primary 58F08, Secondary 58F12, 68Q80.

Research partially supported by N.S.F. 
rather severely restricted by the requirement that they commute with the shift; these constraints are due to the ergodic properties of $\sigma[\mathrm{H} 1-3]$.

In using cellular automata as models, one can view a point $x \in X$ as the assignment of one of a finite number of states-the elements of $S$-to each site on the integer lattice in $\mathbb{R}$. Often one of the states is thought of as being "blank" or "quiescent," while the other states are thought of as "active." In this situation it may be natural to assume on physical grounds that only a finite number of sites are in active states. For definiteness suppose that $S=\{0,1,2, \ldots, N\}$ and let 0 denote the quiescent state. Thus attention can be restricted to the physically relevant subset of $X$,

$$
X_{0}=\{x \in X \mid x(n)=0 \text { for all but finitely many } n \in \mathbb{Z}\},
$$

and to cellular automata that preserve $X_{0} . X_{0}$ is a countable dense subset of $X$ that is invariant under the shift map. It is easy to see that a cellular automaton $f$ on $X$ will map $X_{0}$ into itself if and only if $f(z)=z$, where $z$ is the bisequence consisting entirely of 0 's, $z(n)=0$ for all $n$. This will happen if and only if the generating block map $F$ satisfies $F(0,0,0, \ldots, 0)=0$. For the remainder of the paper we will consider only cellular automata that map $X_{0}$ into itself. This does not seriously limit the range of possible dynamics, for if $f$ is any cellular automaton, then some iterate of $f$ is conjugate to an automaton that preserves $X_{0}$. The reason for this is that $f$ must permute the constant bisequences, so by replacing $f$ with an appropriate iterate and relabeling the symbols we obtain a cellular automaton that has $z$ as a fixed point.

The purpose of this note is to examine the dynamics of restricted cellular automata $f: X_{0} \rightarrow X_{0}$. The main results will show that there are severe constraints on the collections of "attractors" that can occur for a map of this type. These results parallel results obtained in [H1] for unrestricted cellular automata.

References [H2-H3] establish analogous results where the notion of attractor is generalized using shift-invariant probability measures on $X$. Since $X_{0}$ is countable, any probability measure of $X_{0}$ must have an atom; that is, it must assign positive measure to some singleton. If the probability measure is also shift invariant then the orbit of any atom under the shift must be periodic. The only $\sigma$-periodic element of $X_{0}$ is the constant bisequence $z$ consisting entirely of 0 's. Consequently we will consider only topological attractors in this note.

\section{DEFINITIONS AND BACKGROUND}

We continue to assume that $f: X \rightarrow X$ is a cellular automaton that preserves the subset $X_{0}$. In what follows we will be using a metric $d$ on $X$. Any metric that is compatible with the product topology will suffice, for example, $d(x, y)=2^{-n}$, where $n=\min \{\mid j \| x(j) \neq y(j)\}$.

A subset $A$ of $X$ is an attractor of $f$ is there is a nonempty open set $U$ with the two properties: (i) $f(\operatorname{clos}(U)) \subset U$, and (ii) $\bigcap_{n \geq 0} f^{n}(U)=A$. It follows from (i) that $\bigcap_{n \geq 0} f^{n}(U)=\bigcap_{n \geq 0} f^{n}(\operatorname{clos}(U))$, and so the compactness of $X$ ensures that $A$ is compact and nonempty. If the forward orbit of a point $p$ enters $U$, then it stays in $U$ and moves asymptotically toward $A$ under iteration by $f$. The set of all such points is the basin of $A, \mathrm{~B}(A)=$ $\bigcup_{n \geq 0} f^{-n}(U)$. We will refer to a nonempty open set $U$ satisfying (i) as an absorbing set; since $X$ is compact, condition (i) is equivalent to the statement that $U$ is a uniform neighborhood of $f(U)$. 
There will always be at least one attractor, namely $A_{\max }=\bigcap_{n \geq 0} f^{n}(X)$. Any nonempty intersection of finitely many attractors is an attractor. An attractor $A$ is minimal if no proper subset is also an attractor; there may be no minimal attractor. As an example, consider the identity map on $X$. Any nonempty set $U$ that is both open and closed is an attractor for the identity, and since $X$ is a Cantor set, any nonempty open and closed set has a proper subset that is also open and closed.

The following two results can be found in [H1].

1.0. Theorem. Any cellular automaton $f: X \rightarrow X$ is of one of three mutually exclusive types:

(1) $f$ has a minimal attractor. In this case the minimal attractor $A$ is unique, its basin $\mathrm{B}(A)$ is open and dense in $X$, and $A$ shift invariant: $\sigma(A)=A$.

(2) $f$ has a pair of disjoint attractors. In this case each attractor of $f$ contains a pair of disjoint attractors.

(3) $f$ has no minimal attractor, but the intersection of all the attractors of $f$ is nonempty.

1.1. Theorem. If $Y \subset X$ is a finite set that is an attractor for the cellular automaton $f$, then there is a point $p \in Y$ that is fixed by $f$ and is a minimal attractor. Moreover, $p$ is also fixed by the shift (i.e., $p$ is constant as a map from $\mathbb{Z}$ into the symbol set $S$.)

In the next section we will show that these results remain true if $X$ is replaced by $X_{0}$ and $f$ by its restriction to $X_{0}$. Since $X_{0}$ is not compact, it will be necessary to generalize the definition of "attractor." The form of this generalization is primarily motivated by the desire to preserve the connection that exists in the compact case between the collection of attractors of $f$ and the chain recurrent set of $f$. What follows is a brief outline of this connection. A point $p$ is chain recurrent for $f$ if for each $\varepsilon>0$ there is a finite sequence $x_{0}, x_{1}, x_{2}, \ldots, x_{k-1}, x_{k}$ with the property that $d\left(f\left(x_{j}\right), x_{j+1}\right)<\varepsilon$ for $0 \leq j \leq k-1$ and with $x_{0}=x_{k}=p$. This definition is due to Conley, who also noted the following connection between the attractors of $f$ and its chain recurrent set $[\mathrm{C}]$.

1.2. Theorem. Suppose $f$ is a continuous map from a compact space $X$ to itself.

(i) If $A$ is an attractor and $p \in \mathrm{B}(A)-A$, then $p$ is not chain recurrent.

(ii) If $p$ is not chain recurrent, then there is an attractor $A$ with $p \in \mathrm{B}(A)-$ $A$.

The proof of this theorem can be found in [C]; [H1] contains a treatment in the context of cellular automata. Note that the theorem implies that any minimal attractor is contained in the chain recurrent set.

In extending these results to noncompact spaces it is necessary to make some changes in the definitions. In particular, the "attractors" in the compact setting will be replaced by "attractor-like sets." Examples motivating the details of the following definitions can be found in [H4]. 
Definitions. (i) A nonempty open subset $U$ of $X_{0}$ is absorbing for $f$ if there is a constant $\delta>0$ with the property that $U$ contains the $\delta$-neighborhood of $f(U)$.

(ii) If $U$ is absorbing then the set $A=\bigcap_{n \geq 0} \operatorname{clos}\left(f^{n}(U)\right)$ is the attractor-like set determined by $U$. (It is possible that $\bar{A}$ is empty.) The set $\mathrm{B}(A ; U)=$ $\bigcup_{n \geq 0} f^{-n}(U)$ is called the basin of $A$ relative to $U$.

(iii) The extended basin of $A, \mathrm{~B}(A)$, is the union of the sets $\mathrm{B}(A ; U)$ for all absorbing sets $U$ that determine $A$.

In a compact space these definitions reduce to the definitions of absorbing set, attractor, and basin of attraction that were given earlier; see [H4] for details. The form of the definitions is motivated by the desire to obtain the analogue of Conley's theorem in noncompact spaces. The relevant result is

1.3. Theorem. Let $f_{0}: X_{0} \rightarrow X_{0}$ be the restriction of a cellular automaton $f$ to $X_{0}$. Then

(i) If $A$ is an attractor-like set and $p \in \mathrm{B}(A)-A$, then $p$ is not chain recurrent.

(ii) If $p$ is not chain recurrent, then there is an attractor-like set $A$ with $p \in \mathrm{B}(A)-A$.

Proof. In [H4] it is shown that conclusions (i) and (ii) will hold for any continuous map $g$ of a metric space into itself, provided that $g$ satisfies certain mild conditions, for example, if $g$ is either uniformly continuous or a proper map. Since $f_{0}$ is the restriction of a continuous mapping of the compact set $X, f_{0}$ is uniformly continuous.

Theorem 1.3 can be viewed as some evidence that the above definition of attractor-like set is a dynamically natural one.

It is possible for an attractor-like set of the restricted automaton to be empty, even though we insist that absorbing sets be nonempty. This fact will complicate the rest of the paper, but it is a necessary complication. From the point of view of modeling, an attractor-like set that is empty can be viewed as "runaway growth:" the number of sites that are active (nonzero) grows without bound as the automaton is iterated, beginning at appropriate initial points. Examples of such automata are easy to construct.

The following observation will be used in the next section.

1.4. Remark. If $U_{1}$ and $U_{2}$ are absorbing sets, then so is their intersection, provided that it is nonempty.

\section{The MAIN RESUlt}

In this section we will show that the results of Theorem 1.0 carry over to the restricted case. The main result is the following theorem; it is phrased in terms of absorbing sets instead of attractor-like sets because the possiblity that attractor-like sets can be empty means that the notion "minimal attractor-like set" is not a particularly useful one.

2.0. Theorem. Suppose $f_{0}: X_{0} \rightarrow X_{0}$ is the restriction to $X_{0}$ of a cellular automaton $f: X \rightarrow X$. Then $f_{0}$ is of one of the following three mutually exclusive types; type $j$ occurs here precisely when the unrestricted automaton $f$ is of type $j$ in Theorem 1.0. 
(1) There is an absorbing set $W^{*}$ that has the following "minimality" property: if $V$ is any absorbing set then there is an $n \geq 0$ with $f^{n}\left(W^{*}\right) \subset V$. If $A_{0}^{*}$ is the attractor-like set determined by $W^{*}$, then $A_{0}^{*}$ is contained in every attractor-like set of $f, A_{0}^{*}$ is invariant under the shift, and its extended basin is open and dense in $X_{0}$.

(2) There is a pair of disjoint absorbing sets. In this case any absorbing set contains a pair of disjoint absorbing sets.

(3) $f_{0}$ is not of type 1 , but given any finite collection $W_{1}, \ldots, W_{n}$ of absorbing sets, their intersection is nonempty and hence by Remark 1.4 is also absorbing.

Theorem 2.0 will follow from Theorem 1.0 once we make the connection between attractors of $f$ and attractor-like sets of $f_{0}$. Such a connection exists because $X_{0}$ is dense in $X$, and attractors in $X$ are determined by absorbing sets, which are nonempty and open. This makes it possible to set up a correspondence between the absorbing sets of the unrestricted automaton and those of its restriction to $X_{0}$. The proof begins with three simple topological lemmas.

2.1. Lemma. Suppose $(Y, d)$ is a metric space and that $g: Y \rightarrow Y$ is continuous. If $G$ is an absorbing set for $g$ then so is $U=\operatorname{int}(\operatorname{clos}(G))$. Moreover $G$ and $U$ determine the same attractor-like set.

Proof. Clearly $G \subset U \subset \operatorname{clos}(G)$. There is a $\delta>0$ with the property that the distance from $g(y)$ to the complement of $G$ is at least $\delta$ for any $y \in G$, and therefore the same is true for any $y \in U \subset \operatorname{clos}(G)$. If $y \in U$ then $d(f(y), Y-U) \geq d(f(y), Y-G) \geq \delta$, so $U$ is absorbing. The fact that $U$ and $G$ determine the same attractor-like set follows from the fact that $G$ is dense in $U$, so that $\operatorname{clos}\left(g^{n}(G)\right)=\operatorname{clos}\left(g^{n}(U)\right)$ for each $n \geq 1$.

2.2. Lemma. Let $U, G$ be as in the last lemma. Then $\operatorname{int}(\operatorname{clos}(U))=U$.

Proof. We want to show that the two sets $U=\operatorname{int}(\operatorname{clos}(G))$ and $P=$ $\operatorname{int}(\operatorname{clos}(\operatorname{int}(\operatorname{clos}(G))))$ are the same. Working with $P$, if we delete the innermost closure operator we clearly get a subset of $P$; since $G$ is open this new set is equal to $U$. Similarly if we delete the innermost interior operation in $P$ we get a new set that contains $P$, and this new set is also equal to $U$. Thus $P=U$.

In describing the relation between attractors of $f$ on $X$ and attractor-like sets of the restriction of $f$ to $X_{0}$ we will be taking interiors and closures of sets $Y$ with respect to $X$ and to the subspace topology on $X_{0}$. We will denote these operations as $\operatorname{int}(Y ; X), \operatorname{clos}\left(Y ; X_{0}\right)$, etc.

2.3. Lemma. (i) If $Y \subset X_{0}$ then $\operatorname{clos}\left(Y ; X_{0}\right)=X_{0} \cap \operatorname{clos}(Y ; X)$.

(ii) If $Y$ is a closed subset of $X$, then $\operatorname{int}\left(Y \cap X_{0} ; X_{0}\right)=X_{0} \cap \operatorname{int}(Y ; X)$.

Proof. (i) It is clear that the set on the right contains the set on the left. Conversely, there is a closed subset $K$ of $X$ with the property that $\operatorname{clos}\left(Y ; X_{0}\right)=$ $K \cap X_{0}$. In particular $Y \subset K$, so that $\operatorname{clos}(Y ; X)$ is also contained in $K$. Thus

$$
X_{0} \cap \operatorname{clos}(Y ; X) \subset X_{0} \cap K=\operatorname{clos}\left(Y ; X_{0}\right) \text {. }
$$

(ii) The set on the right is open in $X_{0}$ and contained in $Y \cap X_{0}$, so the set on the right is contained in the set on the left. To get the opposite inclusion, let $O$ be an open set in $X$ with the set on the left equal to $X_{0} \cap O$. It suffices to show that $O \subset Y$, for then $O \subset \operatorname{int}(Y ; X)$ and $X_{0} \cap O \subset X_{0} \cap \operatorname{int}(Y ; X)$. 
To see that $O \subset Y$ note that $O \cap X_{0}$ is both dense in $O$ and contained in $Y$ so that $O \subset \operatorname{clos}(O ; X) \subset \operatorname{clos}(Y ; X)=Y$.

2.4. Proposition. Let $f: X \rightarrow X$ be a cellular automaton that maps $X_{0}$ into itself, and let $f_{0}$ denote the restriction of $f$ to $X_{0}$.

2.4.1. If $U$ is an absorbing set for $f$, then $W=U \cap X_{0}$ is an absorbing set for $f_{0}$. If $A, A_{0}$ are the attractor-like sets determined by $U, W$, then $A_{0}=A \cap X_{0}$.

2.4.2. Suppose $V=G \cap X_{0}$ is an absorbing set for $f_{0}$, where $G$ is open in $X$. Let $U=\operatorname{int}(\operatorname{clos}(G))$. Then $U$ is absorbing for $f$, and if $A, A_{0}$ are the attractor-like sets determined by $U, V$, then $A_{0}=A \cap X_{0}$. In particular, the absorbing sets $V$ and $W=U \cap X_{0}$ determine the same attractor-like set of $f_{0}$.

2.4.3. In both 2.4.1 and 2.4.2, the extended basin of $A_{0}, \mathrm{~B}\left(A_{0}\right)$, contains $\mathrm{B}(A) \cap X_{0}$.

Proof of 2.4.1. Clearly if $U$ is absorbing relative to $X$ then $X_{0} \cap U$ is absorbing relative to $X_{0}$. It remains to show that $A_{0}=A \cap X_{0}$. Suppose first that $p \in A_{0}$. Then for each $n \geq 0, p$ is contained in $\operatorname{clos}\left(f^{n}(W) ; X_{0}\right)$. Since $X_{0} \subset X$ and $W \subset U$, we have $\operatorname{clos}\left(f^{n}(W) ; X_{0}\right) \subset \operatorname{clos}\left(f^{n}(U) ; X\right)$. Thus $p$ is contained in $\bigcap_{n \geq 0} \operatorname{clos}\left(f^{n}(U) ; X\right)=A$. Conversely, suppose $p \in A \cap X_{0}$. For each $n \geq 0$

$$
p \in X_{0} \cap \operatorname{clos}\left(f^{n}(U) ; X\right)=X_{0} \cap \operatorname{clos}\left(f^{n}(W) ; X\right)=\operatorname{clos}\left(f^{n}(W) ; X_{0}\right) \text {, }
$$

where the first equality is due to the fact that $W$ is dense in $U$ and the second follows from Lemma 2.3(i). Taking the intersection over all $n \geq 0$ shows that $p \in A_{0}$.

Proof of 2.4.2. Since $X$ is compact, to show $U$ is absorbing we need only verify that $f(\operatorname{clos}(U)) \subset U$. Begin by noting that $V, U$, and $G$ all have the same closures as subsets of $X$. Since $V$ is absorbing for $f_{0}$, there is a positive constant $\delta$ with the property that if $x \in V$ then any point of $X_{0}$ that lies within $\delta$ of $f(x)$ must lie in $V \subset G \subset U$. The density of $X_{0}$ ensures that if $p$ is any point of $X$ that is within $\delta$ of $f(x)$ then $p \in \operatorname{clos}(U)$. The same argument shows that such a $p$ has a neighborhood that is contained in $\operatorname{clos}(U)$. In short, $d(p, f(x))<\delta$ implies that $p \in \operatorname{int}(\operatorname{clos}(U))=U$, where the final equality follows from Lemma 2.2. To finish the proof that $U$ is absorbing for $f$, suppose $y$ is a point of $\operatorname{clos}(U)$. Since $\operatorname{clos}(U)=\operatorname{clos}(V)$, there is a point $x \in V$ with $d(f(x), f(y))<\delta$, and so the preceding argument shows that $f(y) \in U$.

It remains to show that $A_{0}=X_{0} \cap A$. This is complicated by the fact that $G$ might not be absorbing. The argument goes as follows: Lemma 2.1 shows that $V$ and $\operatorname{int}\left(\operatorname{clos}\left(V ; X_{0}\right) ; X_{0}\right)$ are absorbing sets for $f_{0}$ that determine the same attractor-like set, namely, $A_{0}$. On the other hand, 2.4.1 shows that $W=X_{0} \cap U$ is also an absorbing set for $f_{0}$, and that $W$ determines $X_{0} \cap A$. To show that $A_{0}=X_{0} \cap A$ will show that the absorbing sets $\operatorname{int}\left(\operatorname{clos}\left(V ; X_{0}\right) ; X_{0}\right)$ and $X_{0} \cap U$ are equal. Let $Y=\operatorname{clos}(U ; X)$ and observe

$$
\operatorname{clos}\left(V ; X_{0}\right)=X_{0} \cap \operatorname{clos}(V ; X)=X_{0} \cap \operatorname{clos}(U ; X)=X_{0} \cap Y,
$$

where the first equality follows from Lemma 2.3(i) and the second from the fact that $V$ is dense in $U$. It follows that

$$
\operatorname{int}\left(\operatorname{clos}\left(V ; X_{0}\right) ; X_{0}\right)=\operatorname{int}\left(X_{0} \cap Y ; X_{0}\right) \text {. }
$$


Since $Y=\operatorname{clos}(U ; X)$ is closed in $X$, we can apply Lemmas 2.3 (ii) and 2.2 to obtain

$$
\operatorname{int}\left(X_{0} \cap Y ; X_{0}\right)=X_{0} \cap \operatorname{int}(Y ; X)=X_{0} \cap \operatorname{int}(\operatorname{clos}(U ; X) ; X)=X_{0} \cap U .
$$

This completes the proof of 2.4.2.

Proof of 2.4.3. The extended basin of $A_{0}$ is the union of $\mathrm{B}\left(A_{0} ; N\right)$ as $N$ ranges over the collection of absorbing sets that determine $A_{0}$, so it is sufficient to show that $\mathrm{B}\left(A_{0} ; W\right)=\mathrm{B}(A) \cap X_{0} . \mathrm{B}\left(A_{0} ; W\right)$ is defined as $\bigcup_{n \geq 0} f_{0}^{-n}(W)$, and $f_{0}^{-n}(W)=f^{-n}(W) \cap X_{0}=f^{-n}\left(U \cap X_{0}\right) \cap X_{0}$. Since $X_{0}$ is forward invariant under $f$, this last set is the same as $f^{-n}(U) \cap X_{0}$. The union of the sets $f^{-n}(U) \cap X_{0}$ is $\mathrm{B}(A) \cap X_{0}$.

We will refer to the following consequence of the proof of 2.4.2: for $V, U$ as in 2.4.2, we have

$$
V \subset U \cap X_{0} \subset \operatorname{clos}\left(V ; X_{0}\right) .
$$

The first inclusion is obvious and the second follows from $(*)$.

Proof of Theorem 2.0. We will show that for $j=1,2,3$, if the unrestricted automaton $f$ is of type $j$ in Theorem 1.0, then its restriction is of type $j$ in Theorem 2.0.

(1) Let $A^{*}$ be the minimal attractor of $f$ and let $U^{*}$ be an absorbing set that determines $A^{*}$. Set $W^{*}=U^{*} \cap X_{0}$, and denote the attractor-like set determined by $W^{*}$ by $A_{0}^{*}$. Suppose $V$ is some absorbing set for $f_{0}$, and as in 2.4.2 let $U$ be the corresponding absorbing set for $f$. If $A$ is the attractor for $f$ determined by $U$, then $A^{*} \subset A \subset U$. Since $X$ is compact and $A^{*}=$ $\bigcap_{n \geq 0} f^{n}\left(U^{*}\right)$, there is an integer $n$ with $f^{n}\left(U^{*}\right) \subset U$. Thus

$$
f^{n}\left(W^{*}\right) \subset f^{n}\left(U^{*}\right) \cap X_{0} \subset U \cap X_{0} .
$$

Combining this with $(2.5)$ we obtain

$$
f^{n+1}\left(W^{*}\right) \subset f\left(U \cap X_{0}\right) \subset f_{0}\left(\operatorname{clos}\left(V ; X_{0}\right)\right) \subset V,
$$

where we use the assumption that $V$ is absorbing for $f_{0}$ to obtain the last inclusion. Since the basin of $A^{*}$ is open and dense in $X$, the extended basin of $A_{0}^{*}$ will be open and dense in $X_{0}$ by 2.4.3. $A_{0}^{*}$ is shift invariant because $A_{0}^{*}$ is the intersection of $A^{*}$ and $X_{0}$, both of which are shift invariant.

(2) Let $A_{1}, A_{2}$ be a disjoint pair of attractors for $f$, and let $U_{1}, U_{2}$ be associated absorbing sets. Since $X$ is compact, the forward orbit of any point in the closure of $U_{i}$ tends asymptotically toward $A_{i}$, so the closures of $U_{1}$ and $U_{2}$ must be disjoint. Let $V_{i}=U_{i} \cap X_{0}$ be the associated absorbing sets for $f_{0}$. $V_{1}$ and $V_{2}$ also have disjoint closures, so they determine disjoint attractor-like sets. This gives the first assertion of (2). To establish the second, consider some absorbing set $V$ for $f_{0}$. Let $G, A$ and $U$ be as in 2.4.2. $A$ is an attractor for $f$, so $A$ contains a disjoint pair of attractors. Due to the compactness of $X$, this means that a disjoint pair of absorbing sets can be found in any given neighborhood of $A$; in particular, they can be found in $U$; call them $U_{1}$ and $U_{2}$. As above, if $V_{i}=U_{i} \cap X_{0}$ then $V_{1}$ and $V_{2}$ are disjoint absorbing sets for $f_{0}$. By Remark 1.4 the sets $V_{i} \cap V$ are absorbing if they are nonempty, so to finish the proof of (2) it is enough to show that $V \cap V_{i} \neq \varnothing$ for $i=1,2$. To 
do this, recall that $G$ is open in $X$ with $V=G \cap X_{0}$ and $U=\operatorname{int}(\operatorname{clos}(G))$. Thus $G$ is open and dense in $U$, so $G \cap U_{i}$ is open and nonempty. Since $X_{0}$ is dense in $X$, we conclude that $\varnothing \neq G \cap U_{i} \cap X_{0}=\left(G \cap X_{0}\right) \cap\left(U_{i} \cap X_{0}\right)=V \cap V_{i}$.

(3) In this case $f$ has no minimal attractor, but all the attractors of $f$ intesect. First we show that if $V_{1}, V_{2}$ are absorbing sets for $f_{0}$, then $V_{1} \cap V_{2} \neq \varnothing$. For $i=1,2$ let $G_{i}$ and $U_{i}$ be the open subsets of $X$ associated to $V_{i}$ as in 2.4.2. Each $U_{i}$ is absorbing for $f$ and so determines an attractor. These attractors intersect, so the open set $U=U_{1} \cap U_{2}$ is nonempty. $U_{i}=\operatorname{int}\left(\operatorname{clos}\left(G_{i}\right)\right.$, so $G_{i}$ is an open and dense subset of $U_{i}$, and thus $G_{1} \cap G_{2}$ is open and dense in $U$. In particular, $G_{1} \cap G_{2}$ is nonempty; since it is open, its intersection with $X_{0}$ is also nonempty. Thus $V_{1} \cap V_{2}=G_{1} \cap G_{2} \cap X_{0} \neq \varnothing$. It remains to show that $f_{0}$ is not of type (1); to do this we will show that if $V$ is any absorbing set for $f_{0}$ then there is another absorbing set $W$ with $f^{n}(V)$ not contained in $W$ for any $n$. Given $V$, let $U$ be absorbing for $f$ as in 2.4.2. Since the attractor determined by $U$ is not minimal, there is another absorbing set for $f, N$, such that $N$ does not contain $f^{n}(U)$ for any $n$. Let $W=N \cap X_{0}$. If $f^{n}(V) \subset W$, then

$$
f^{n}(U) \subset \operatorname{clos}\left(f^{n}(U)\right)=\operatorname{clos}\left(f^{n}(V)\right) \subset \operatorname{clos}(W) \subset \operatorname{clos}(N)
$$

where the closures are taken in $X$, and we have used the density of $V$ in $U$. Applying $f$ and using the assumption that $N$ is absorbing gives the contradiction $f^{n+1}(U) \subset N$.

2.6. Corollary. Using the notation of Theorem 2.0, suppose $f_{0}$ is a type 1 cellular automaton and that $A_{0}^{*}$ is finite. Then either $A_{0}^{*}$ is the empty set or else $A_{0}^{*}=\{z\}$ where $z$ in $X$ is the configuration that has the quiescent state at every site: $z(n)=0$ for all $n \in \mathbb{Z}$.

Proof. The theorem shows that $A_{0}^{*}$ is shift-invariant; since it is also finite, each point in $A_{0}^{*}$ must be periodic under the shift. If $x \in A_{0}^{*}$ is nonzero at some site $n$ it will be nonzero at infinitely many sites, which contradicts the fact that $x \in X_{0}$.

Corollary 2.6 can be interpreted as saying that the only finite minimal attractor-like sets for a restricted cellular automaton correspond either to runaway growth for almost all initial conditions or to eventual extinction for almost all initial conditions.

\section{3. n-DIMENSIONAL CELLULAR AUTOMATA}

Strictly speaking the cellular automata described above are one-dimensional cellular automata. For each finite $n$ there is a related concept of $n$-dimensional cellular automata. These are defined as follows: let $S$ continue to denote a finite set of symbols, and let $X$ be all maps from the integer lattice in $\mathbb{R}^{n}$ to $S, X=\left\{x: \mathbb{Z}^{n} \rightarrow S\right\}$. For each $w$ in $\mathbb{Z}^{n}$ there is an associated shift on $X, \sigma_{w}: X \rightarrow X$, defined as $\left(\sigma_{w} x\right)(v)=x(v+w)$. An $n$-dimensional cellular automaton is a map $f: X \rightarrow X$ that commutes with all of these shifts.

Theorems 1.0 and 1.2 are true for $n$-dimensional cellular automata; see [H1]. The argument in $\S 2$ that derives Theorem 2.0 from Theorem 1.0 does not require that the cellular automata being considered to one-dimensional, so Theorem 2.0 and Corollary 2.6 also hold for any finite-dimensional cellular automaton. 


\section{REFERENCES}

[C] C. Conley, Isolated invariant sets and the Morse index, CBMS, vol. 38, Amer. Math. Soc., Providence, RI, 1978.

[CHY] K. Culik II, L. P. Hurd, and S. Yu, Computation theoretic aspects of cellular automata, Phys. D 46 (1990), 357-378.

[FTS] D. Farmer, T. Toffoli, and S. Wolfram (eds.), Cellular automata: proceedings of an interdisciplinary workshop, Phys. D 10 (1984).

[Hed] Hedlund, Endomorphisms and automorphisms of the shift dynamical system, Math. Systems Theory 3 (1969), 320-375.

[H1] M. Hurley, Attractors in cellular automata, Ergodic Theory and Dynamical Systems 10 (1990), 131-140.

[H2] _ Ergodic aspects of cellular automata, Ergodic Theory and Dynamical Systems 10 (1990), 671-685.

[H3] - Varieties of periodic attractor in cellular automata, Trans. of the AMS, 326 (1991), 701-726.

[H4] _ Chain recurrence and attraction in noncompact spaces, Ergodic Theory and Dynamical Systems, to appear.

[W] S. Wolfram, Theory and applications of cellular automata, World Scientific, Singapore, 1986.

Department of Mathematics and Statistics, Case Western Reserve University, Cleveland, Ohio 44106-7058

E-mail address:mgh3@po.CWRU.EDU 